\title{
Non-destructive control data analysis of railroad foundation constructions
}

\author{
Alexander Trofimov ${ }^{1, *}$, Albina Kuzmenko ${ }^{1}$, Halyna Nesterenko ${ }^{2}$, Svitlana Avramenko ${ }^{3}$, Mykhailo Muzykin ${ }^{3}$, Nikolaj \\ Mormul $^{4}$, and Anatoliy Sokhatsky ${ }^{5}$ \\ ${ }^{1}$ UCF, Department of Transportation systems and technologies, 49000, Dnipro, Volodymyra Vernadskoho str., 2/4, Ukraine \\ ${ }^{2}$ DNURT, Department of Operational Work Management, 49010, Dnipro, Lazaryan str., 2, Ukraine \\ ${ }^{3}$ DNURT, Department of Life Activity Safety, 49010, Dnipro, Lazaryan str., 2, Ukraine \\ ${ }^{4}$ UCF, Department of Applied Mathematics and Informatics, 49000, Dnipro, Volodymyra Vernadskoho str., 2/4, Ukraine \\ ${ }^{5}$ Institute of transport system and technologies of National academy of sciences of Ukraine, Department 7, 49005, Dnipro, \\ Pisargevskoho str., 5, Ukraine
}

\begin{abstract}
The evaluation of the parameters of multi-layered foundations (railroad basis, foundations of railway structures, etc.) plays an important role in ensuring the safe movement of trains. The method of estimating the mechanical and geometric parameters of such foundations based on the solutions of inverse problems for multi-layered elastic packets is proposed. As input data for such problems the measured displacements of certain points on the package surface are used. The method allows estimating the parameters of the a priori distribution of unknown variable parameters, identifying and excluding outliers of the measured data from the created model, and constructing a posteriori estimation of the unknown parameters probability density with acceptable resolution. Proposed method can be used to create a new generation of equipment intended for non-destructive monitoring and estimating of the condition of the railroad basis and the foundations of artificial structures. The appropriate software of such vehicles based on the developed methods of data processing can be developed. The use of such equipment allows to operatively analyzing the state of individual areas of the railroad to decide on the need of repairing or replacing the railroad base or foundation of other elements of railroad infrastructure.
\end{abstract}

\section{Introduction}

Problem statement. The issues of non-destructive monitoring and control of the mechanical and geometrical parameters of the roadbed are of great importance in the practice of safe railways operation. The uncertainty of the train movement process over long distances greatly complicates the transportation planning and ensuring traffic safety. This is due to factors associated with the risks in the process of organizing the movement of trains, when it is necessary to ensure high reliability of all railway subsystems, in particular infrastructure subsystems. Therefore, the evaluation of the parameters of multi-layered foundations (railways, foundations of railway structures, etc.) plays an important role in ensuring the safety of trains [1]. It is known that the consequences of train derailments caused by the collapse of railway embankments often exceed the consequences of accidents caused by the destruction of the rail track itself [2]. In addition, the cost of restoring the damaged subgrade and associated infrastructure is usually significantly higher. This raises the problem of creating methods that are more accurate for analyzing the diagnostic parameters of the roadbed structures to predict possible unallowable deformations and/or damage.

Review of resent researches and publications. There is a variety of works devoted to roadbed state diagnostics. For example, E.V. Nepomnyashchikh and K.A. Kirpichnikov in [3] set the tasks of diagnostics of the roadbed, namely: determining the parameters, that define the deformation properties of the roadbed; predicting the values of such parameters in time and their influence on the deformations occurrence of the roadbed construction; development of new principles, methods and technical means of diagnosing in order to detect "bottlenecks" in such structures. V.D. Petrenko, D.O. Yampolsky, I.O. Svyatko devoted his work [4] to the analysis of the existing modern and classical methods of numerical modeling of behavior of soil samples under the action of a static load. They proposed a new computational model, in which, in addition to the classical approach to the analysis of the stress state of the roadbed soil, the deformation state is taken into account. In the article [5] by scientists Luchko Y.Y. and Kravts, I. B., the condition of the roadbed on the railways of Ukraine is analyzed, the requirements for it and the methods of monitoring are considered. The attention is focused on the importance of monitoring the state of

Corresponding author: atrof2222@gmail.com 
railway infrastructure facilities in the context of introducing high-speed traffic on the Ukrainian railways. The ground penetrating radar method (GPR) as one of the most promising methods for monitoring of the roadbed is proposed. The world and domestic experience in the use of GPR for non-destructive monitoring of the condition of the roadbed, ballast layer and artificial structures is also given. Its advantages are efficiency of work and low labor intensity. However, the methods proposed in this work provide information only about the geometrical parameters of the railroad bed base and do not give any information of its mechanical properties.

The purpose of the article. The main goal of this work is to develop methods for analyzing data from scanning devices designed to determine the surface points displacement velocities of the loaded railway bed, which helps to recognize the weakness of the roadbed structures and prevent unallowable deformations and destruction of the it and, thus, increase the safety of train traffic.

\section{Analysis of methods and technologies for determining the diagnostic parameters of the roadbed}

The diagnostic parameters for assessing the condition of the roadbed can be divided into three groups: geometric (the shape and size of the soil base layers that make up the roadbed); mechanical (elastic deformation moduli, Poisson's ratios, elastic wave propagation velocities, plasticity, creep parameters, etc. of base layer materials); physical (indices of electrical and thermal conductivities, conductivity parameters of penetrating radiation, characteristics of moisture saturation, porosity, etc.). The most important in terms of assessing the deformation properties of the soil base are the first two groups of diagnostic parameters. Physical parameters mainly serve to estimate more accurately the geometric and mechanical characteristics.

For obtaining the diagnostic parameters one use the following methods.

1. Engineering and geodetic. They include the scanning of transverse embankment profiles, the binding of boreholes and geophysical profiles.

2. GPR. The method is based on determining the geological characteristics of the soil foundation using the parameters of short high-frequency electromagnetic pulses from the generator, transmitted and received through antennas located on the ground surface. Devices that allow such sounding are called ground penetrating radars. By ground penetrating radars it is possible to determine the geometrical characteristics of material layers that make up the soil foundation. One can also estimate some of the physical characteristics of the layers (moisture saturation, porosity, etc.). GPR techniques are also adjacent to the methods of electrodynamic sensing.

3. Method of loading trains. It consists in the determination of the elastic sediment of the railway track under the action of a passing loading train [3].
4. Method of measuring the surface points elastic displacement of the roadbed under the passing trains. The method is based on measuring the amplitude of the oscillation of the roadbed surface under the action of passing trains. Measurements are carried out using lowfrequency sensors installed at various points on the subgrade surface.

5. Seismic method. In the seismic method, the velocities of elastic waves excited in the ground bed by a shock load are recorded. Taking into account this information, the characteristics of the velocities of propagation of shock waves and their amplitudefrequency parameters in the soil massif are investigated, which makes it possible to draw conclusions about the structure of the subgrade.

Technologically diagnostic examination is carried out in two stages. At the preliminary stage, "problematic" sections of the roadbed are identified, which are then subjected to more thorough examination at the main stage (detailed inspection stage).

Requirements for the preliminary stage are high survey speed, low cost, the possibility of collecting as much data as possible for preliminary analysis. The procedures of the main stage (seismic sounding, measurements using low-frequency sensors) are usually much more expensive and time-consuming.

The main tools of the preliminary stage of diagnostics are usually GPR sounding and the use of loading trains. The main disadvantage of such an organization is that georadars are generally not useful for assessing the mechanical properties of the investigated subgrade layer materials, and information from load trains (deformation of the track under load) is usually insufficient for adequately estimation of the entire layered foundation deformation properties. The procedures of the main stage of diagnosis are quite informative, but they are expensive and have a longer duration in time. At the same time, the existing level of development of measuring devices and data processing methods makes it possible to create diagnostic tools that combine high data acquisition rates with sufficient coverage of the most "informative" part of the deformable foundation.

Such diagnostic tools, that allows to obtain the small displacement (or velocity) of deformable surface points, call "deflectometers". Modern deflectometers are already widely used in the practice of roads building and operating for diagnosing the condition of the pavement $[6,7]$. Such devices are divided into two classes. Devices of the first class allow estimating the displacement of the test surface points. They can be called "displacement deflectometers". Such tools use, as a rule, optical sensors-rangefinders [11], mounted on the bottom of the body of a special vehicle that creates the necessary loading of the diagnosed pavement. The disadvantage of such tools is that they must be equipped with a complex system of precise positioning of rangefinder sensors, as well as a speed integrator to determine accurately the time intervals between fixing the results of two adjacent displacement sensors at the same point of the investigated surface. 
Another significant problem is the vibration of the mounting base of optical sensors, the amplitude of which can significantly (sometimes by several orders of magnitude) exceed the values of the measured displacements. This problem may partly be solved by installing damping devices, as well as accelerometersintegrators, which take into account the vertical and horizontal movements of the sensor mounting base.

Deflectometers of the second type, called "velocity deflectometers", lack these deficiencies [8]. They use optical Doppler sensors to determine the velocities of certain points of the investigated surface, which serve as the initial data for calculating the parameters of the roadbed. As a rule, such instruments are also equipped with rangefinder sensors for simultaneous scanning of the surface shape of the foundation under study. Such combined tools can be installed on the locomotive (see Fig. 1) and used to scan the interesting parameters of the train roadbed construction in online mode with high speed. This approach will provide the minimum amount of time and money without changing the train schedule.

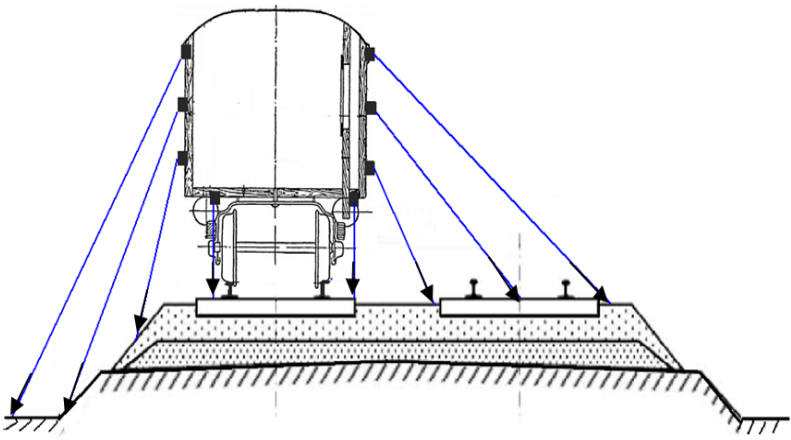

Fig. 1. The scanning of the roadbed by deflectometers installed on the locomotive.

It is possible to install deflectometers at different angles to the testing surface, which will increase the measurement flexibility, making it possible to focus the scan on the most informative part of the deformable foundation.

At the present stage of development of testing devices, dynamic deflectometers of the considered types are equipped with a number of scanning sensors of some units [6], which is clearly not enough to obtain any detailed information about the parameters of the testing multi-layered subgrade. As a rule, the use of such devices consists in finding a certain integral index (for example, SNP, Adjusted Structural Number, [9]) by means of certain empirical formulas, which characterizes the residual strength of the testing structure. At the same time, the existing development of hardware measurement tools, as well as progress in the development of effective data processing methods, allows using a larger number of input data (moreover, data from both deflectometer sensors and georadar and other devices), which, obviously, will make it possible to estimate more parameters of layered foundation, and perhaps with greater accuracy. This will allow to create a new generation high-speed tools for monitoring the state of the roadbed with online interpretation of obtained measurements.

Data processing supplied by the considered tools is based on certain models of layered foundations, discussed below.

\section{Models of layered foundations}

Parameters taking into account during the modeling of layered foundations can be divided into such main groups (Fig. 2).

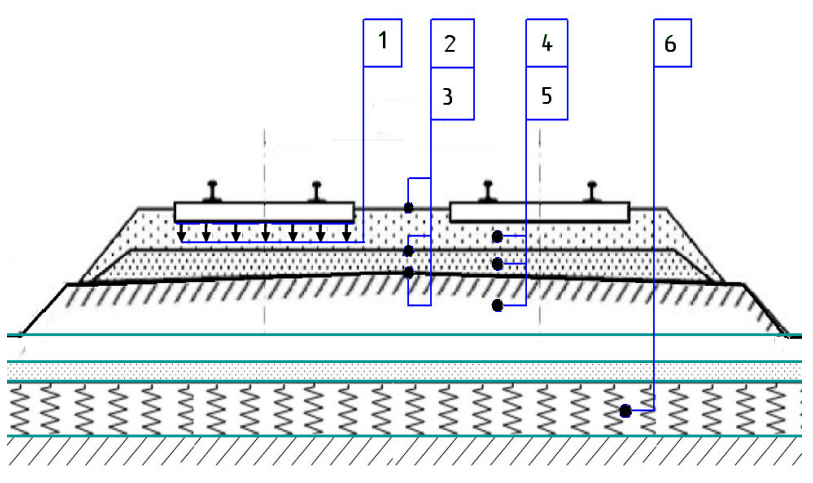

Fig 2. Layered foundation model.

1. Parameters of the distributed load that this layered packet is subjected to. Define the spline or other approximating function.

2. Characteristics of layered packet geometry. This group of parameters determines the number and thickness of the layers, the forms of the boundary between layers and top free surface defined by splines or other approximating functions.

3. Properties of the interaction between layers. These are type of the interaction between layers (hard grip or the possibility of slipping according to Amontons-Coulomb law etc.) and its parameters.

4. Mechanical properties of the packet layers. These, primarily, the elasticity constants (for example, shear modulus $G$ and Poisson's ratio $v$ ), parameters characterizing plastic, viscoelastic etc. properties of layers material and also fatigue development rate.

5. Other physical parameters of layers material. These include degree of compaction, porosity, permeability, viscosity etc.

6. Properties of the ground subgrade where the considered packet is located on. Fig. 2 shows the Pasternak's subgrade model that is described by two parameters: shear modulus $G_{S}$ and "spring" stiffness rate $k$, but one can use models with less (Winkler foundation model) of more (Kerr subgrade model) parameters, however.

All or some of these properties (include discretized version of mentioned functions) form a model parameters vector $\mathbf{m}=\left(\mathrm{m}_{1}, \mathrm{~m}_{2}, \ldots\right)^{\mathrm{T}}$. Mathematical model of layered foundation formulated in displacement velocities can be expressed as an operator equation

$$
\mathbf{A}[\mathbf{m}](\mathbf{v})=\dot{\mathbf{f}}[\mathbf{m}] .
$$


Here $\mathbf{v}=\mathbf{v}(\mathbf{x})=\dot{\mathbf{u}}$ is a vector-function of displacement velocities of packet points (point over symbol means as usual time derivative) defined in composite domain $\Omega=\Omega_{1} \cup \Omega_{2} \cup \ldots \cup \Omega_{\mathrm{K}}\left(\Omega_{i}, i=\overline{1, K}\right.$ - domain, holds by the $i$-th layer of the packet, $K$-th layer is in the bottom of packet), $\mathbf{f}[\mathbf{m}](\mathbf{x})$ - volumetric forces vector, $\mathbf{A}[\mathbf{m}](\cdot)$ : $\mathrm{H}_{\mathbf{v}}[\mathbf{m}] \rightarrow \mathrm{H}_{\mathbf{f}}[\mathbf{m}]-$ vector operator, which components defined as

$$
\mathrm{A}_{i}[\mathbf{m}](\mathbf{v})=\frac{\partial \dot{\sigma}_{i j}[\mathbf{m}]\left(\varepsilon_{k l}(\mathbf{v})\right)}{\partial x_{j}}, \quad i, j, k, l=1,2,3
$$

We use the summation over repeated indexes as is customary in tensor analysis. Components of stress velocities tensor $\dot{\sigma}$ relates with small deformation velocity tensor components $\dot{\varepsilon}$ by the defining relations (fundamental law of material)

$$
\dot{\sigma}_{i j}=\dot{\sigma}_{i j}[\mathbf{m}]\left(\dot{\varepsilon}_{k l}\right)
$$

It can be linear elastic law, Prandtl-Reuss plastic flow equations or other more sophisticated plasticity (or viscoelasticity) theory equations. Small deformation velocity tensor components defines through the displacement velocities by Cauchy-Green formulas

$$
\dot{\varepsilon}_{k l}(\mathbf{v})=\frac{1}{2}\left(\frac{\partial \mathrm{v}_{k}}{\partial x_{l}}+\frac{\partial \mathrm{v}_{l}}{\partial x_{k}}\right)
$$

Structure of functional space $\mathrm{H}_{\mathrm{v}}[\mathbf{m}]$ of displacement velocities $\mathbf{v}=\mathbf{v}(\mathbf{x})$ defines by the system of restrictions: boundary conditions

$$
\begin{array}{r}
\dot{\sigma}_{n}(\mathbf{x})=\dot{\mathbf{T}}[\mathbf{m}](\mathbf{x})=\left(\dot{\mathrm{T}}_{1}(\mathbf{x}), \dot{\mathrm{T}}_{2}(\mathbf{x}), \dot{\mathrm{T}}_{3}(\mathbf{x})\right)^{\mathrm{T}}, \\
\mathbf{x} \in \mathrm{S}_{\sigma}, i=1,2,3, \\
\mathbf{v}(\mathbf{x})=\mathbf{v}_{\mathrm{u}}[\mathbf{m}](\mathbf{x}), \mathbf{x} \in \mathrm{S}_{\mathrm{u}}, \mathrm{S}=\partial \Omega=\mathrm{S}_{\sigma} \cup \mathrm{S}_{\mathrm{u}},
\end{array}
$$

(n is outer normal unit vector to the surface of the packet, $\sigma_{n i}=\sigma_{i j} n_{j}$ - coordinates of normal component of the stress vector) and condition of interaction between packet layers and between the packet and the subgrade. In case of hard concatenation («gluing») of layers $i$ and $k$ on the contact surface $\mathrm{S}_{i k}[\mathbf{m}]=\partial \Omega_{i} \cap \partial \Omega_{k}$ we have

$$
\mathbf{v}^{(i)}(\mathbf{x})=\mathbf{v}^{(k)}(\mathbf{x}), \mathbf{x} \in \mathrm{S}_{i k}
$$

in case of contact with slipping of scabrous layers according to Amontons-Coulomb friction model

$$
\left\{\begin{array}{l}
\mathrm{v}_{n}{ }^{(i)}(\mathbf{x})=\mathrm{v}_{n}{ }^{(k)}(\mathbf{x}), \\
\dot{\sigma}_{\tau}{ }^{(i)}(\mathbf{x})=\mu_{i k}[\mathbf{m}](\mathbf{x}) \dot{\sigma}_{n}{ }^{(k)}(\mathbf{x}),
\end{array}, \mathbf{x} \in \mathrm{S}_{i k},\right.
$$

where $\mathbf{n}$ is normal vector to the contact surface $S_{i k}, \tau \perp \mathbf{n}$ is unit tangent vector determined as

$$
\begin{gathered}
\tau(\mathbf{x})=\frac{\mathbf{V}_{\tau}{ }^{(k)}(\mathbf{x})-\mathbf{V}_{\tau}{ }^{(i)}(\mathbf{x})}{\left|\mathbf{V}_{\tau}{ }^{(k)}(\mathbf{x})-\mathbf{V}_{\tau}{ }^{(i)}(\mathbf{x})\right|}, \mathbf{v}_{\tau}{ }^{(i)}=\mathbf{v}^{(i)}-\mathbf{v}_{n}{ }^{(i)}, \\
\mathbf{V}_{\tau}{ }^{(k)}=\mathbf{v}^{(k)}-\mathbf{v}_{n}{ }^{(k)}, \mathbf{x} \in \mathrm{S}_{i k},
\end{gathered}
$$

$\mu_{i k}=\mu_{i k}[\mathbf{m}](\mathbf{x})$ is friction coefficient between layers $i$ and $k$.

Winkler model of packet interaction with the subgrade defines by the relation (we neglect friction between $K$-th packet layer and the subgrade)

$$
\left\{\begin{array}{l}
\dot{\sigma}_{n}{ }^{(K)}(\mathbf{x})=k_{\mathrm{S}}[\mathbf{m}](\mathbf{x}) \mathrm{v}_{n}{ }^{(k)}(\mathbf{x}), \\
\dot{\sigma}_{\tau}{ }^{(K)}(\mathbf{x})=0
\end{array}\right.
$$

where $\mathrm{S}_{K}$ is the contact surface of the $K$-th layer and the subgrade, $k_{\mathrm{S}}[\mathbf{m}](\mathbf{x})$ is the local stiffness coefficient of the subgrade.

Equation (1) forms so-called "forward problem": having certain parameters vector $\mathbf{m}$ one needs to obtain the vector of displacement velocity $\mathbf{v}(\mathbf{x})$

$$
\mathbf{v}=\mathfrak{R}(\mathbf{m}),
$$

where $\mathfrak{R}(\cdot): \mathbf{H}_{\mathbf{M}} \rightarrow \mathbf{H}_{\mathbf{v}}[\mathbf{m}]$ is a forward operator, $\mathbf{H}_{\mathbf{M}}$ is model parameters vector space. For an "inverse problem" formulation one should takes into account an operator $\aleph(\cdot): \mathbf{H}_{\mathbf{v}}[\mathbf{m}] \rightarrow \mathbf{H}_{\mathrm{D}}$, that for each $\mathbf{v}(\mathbf{x})$ defines a "model data vector" of the problem

$$
\mathbf{d}=\aleph(\mathbf{v})=\aleph(\Re(\mathbf{m}))=\mathbf{g}(\mathbf{m}),
$$

where $\mathbf{H}_{\mathbf{D}}$ is a model data space, $\mathbf{g}(\cdot): \mathbf{H}_{\mathbf{M}} \rightarrow \mathbf{H}_{\mathbf{D}}$ is a "forward problem operator". As a model data, one should consider a radial velocity $\mathbf{v}_{i}$ of certain roadbed point $\mathrm{A}_{i}$, scanned by each used deflectometer (Fig. 1):

$$
\mathrm{d}_{i}=l_{i j} \mathrm{v}_{i j}, j=1,2,3, \quad i=1,2, \ldots, K_{d},
$$

where $l_{i j}$ are direction cosines of $i$-th deflectometer scanning ray, $K_{d}$ is number of deflectometers used.

The essence of inverse problem is "to invert" the forward problem operator $\mathbf{g}(\cdot)$ : for each (may be noisy) measured by the deflectometers vector $\mathbf{d}$ find the corresponding model parameters vector $\mathbf{m}$, that satisfies (12).

Method for solving the formulated inverse problem is proposed in [13]. This method is based on informationprobabilistic (Bayesian) approach to solving the inverse problem [12]. According to this approach, solution of this problem is a posterior probability density of estimated model parameters vector $\mathbf{m}$. For this approach, the "prior" (or "a priori") information about estimated parameters $\mathbf{m}$, expressed as a correspondent prior probability density, plays a very important role. Without such information sometimes is impossible to obtain the qualitative inverse problem solution.

\section{Multigrid solutions of the forward problem}

To investigate the solutions of the considered inverse problems for a layered foundation, gradient methods to minimize a certain residual functional, and statistical modeling methods (Monte-Carlo) to study the behavior of this functional near extreme points are used. When using any of these methods, the key issue is the effective solution of the "direct problem" for layered packet with 
specified characteristics defined by a certain (fixed) model parameters vector $\mathbf{m}$. For this purpose, one should solve two such main associated issues.

1. Choose an appropriate discretization scheme.

2. Develop fast numerical algorithm for solving obtained system of grid equations.

The choice of a successful discretization scheme determines the accuracy of the obtained solution of a differential problem (1). For both the most popular Finite Element and Finite Difference Methods the building of appropriate computational grid defines the quality of entire scheme of discretization. In [14] the problem of creating practical grid for an elastic layered packet was considered. The recommendations are: 1) use a regular grids as the most suitable for further calculations; 2) use interpolation formulas as grid construction procedures (Fig. 3).

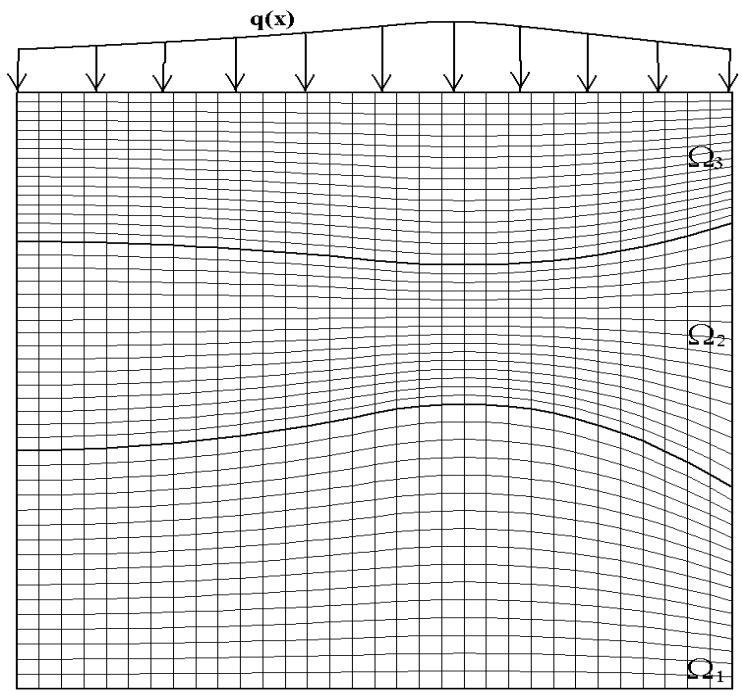

Fig. 3. Regular grid created for the layered packet by interpolation formulas technique.

As a result of the discretization process we obtain the system of algebraic equations

$$
\mathbf{A}^{\mathbf{h}} \mathbf{v}^{\mathbf{h}}=\mathbf{f}^{\mathbf{h}}
$$

(h is a discretization parameter), that approximates the source differential problem (1). The main task is to effectively (numerically) solve the system (14).

Today one of the most advanced for such purpose (especially for elliptic problems like (1)) is a Multigrid Method [15], first proposed by R.P. Fedorenko in 1962. It's efficiency is defined by the fact, that it needs $\mathrm{O}(N)$ arithmetic operations to approximately solve (14) "at the level of discretization error", where $N$ is the number of unknowns in the system (14). This method is based (in its classical Fedorenko's formulation) on two main ideas. First, instead of solving the system (14) on only one grid, one considers the discretization of the problem (1) on the system of nested grids that forms a "hierarchy of grid levels". So the discretized problems

$$
\mathbf{A}_{k} \mathbf{v}_{k}=\mathbf{f}_{k}, \quad k=1,2 \ldots, K_{\max }
$$

are considered, where the index $k=0$ (the lowest grid level) corresponds the coarsest grid and the system (15) contains a little number of unknowns, that provides the fast solving of it by a direct method (Gauss or Cholesky decomposition). Index number $k=K_{\max }$ (the highest grid level) corresponds the finest grid (grid with the smallest mesh-size), that is our goal for system (14) to solve.

The solving procedure begins from the lowest grid level $k=0$. Then obtained solution interpolates to the higher level $k=1$, where it is refining, and then corrected solution interpolates to the next level $k=2$ and so on. This procedure makes up the essence of so-called Full Multigrid (FMG) Algorithm.

The method of précising the approximate solution on the level $k$ is the most sophisticated issue in entire multigrid theory. The main idea of this method is to use the means of coarser grids for correction of the solution on the current level $k$. This procedure calls Coarse Grid Correction (CGC) Algorithm or CGC-Cycle.

Algorithm CGC_CYCLE $\left(k, \mathbf{f}_{k}, \mathbf{u}_{k}^{(0)}, v_{1}, v_{2}, \gamma\right)$

${ }^{*}$ Algorithm for approximate solution of a system of linear equations $\mathbf{A}_{k} \mathbf{v}_{k}=\mathbf{f}_{\mathbf{k}}$, where $k$ is current grid level, $\mathbf{v}_{k}^{(0)}$ is initial approximation, $\mathbf{A}_{k}$ is system matrix, $\mathbf{f}_{k}$ is right-hand side, $v_{l}$ is number of pre-iteration of relaxation scheme, $v_{2}$ is number of post-iterations, $\gamma$ is a cycle parameter, $\mathbf{v}_{k}^{(l)}$ is refined approximate solution. */

\section{Begin}

1. If $k=0$, then the system (15) is solved by direct method. As a result, one obtains the value $\mathbf{v}_{\mathbf{k}}^{(1)}$. Go to step 9.

2. (Pre-relaxation). Perform $v_{1}$ smoothing iterations by the formula

$$
\widetilde{\mathbf{V}}_{k}^{(0)} \leftarrow \mathbf{S}_{k}^{v_{1}} \mathbf{V}_{k}^{(0)}
$$

where $\mathrm{S}_{k}$ is a relaxation scheme operator.

3. Calculate the residuals by

$$
\mathbf{r}^{\mathrm{k}} \leftarrow \mathbf{A}_{k} \widetilde{\mathbf{v}}_{k}^{(0)}-\mathbf{f}_{k} .
$$

4. Projection of the residuals to the coarser grid (level $k-1)$

$$
\mathbf{r}^{k-1} \leftarrow \mathrm{R}_{k}^{k-1} \mathbf{r}^{k}
$$

where $\mathrm{R}_{k}^{k-1}$ is a projection operator from finer to coarser grid.

5. Calculation and refinement of the correction vector $\mathbf{e}^{\mathrm{k}-1}$ on the coarser grid, that is solving the coarse grid equation

$$
\mathbf{A}_{k-l} \mathbf{e}^{k-1}=\mathbf{r}^{k-l}
$$

recursively by the same algorithm, beginning with null initial approximation

$$
\begin{aligned}
& \widetilde{\mathbf{e}}_{k}^{(0)} \leftarrow \mathbf{0} \\
& \text { For } i \leftarrow 1 \text { to } \gamma \text { do } \\
& \widetilde{\mathbf{e}}_{k-1}^{(i)} \leftarrow \text { CGC_CYCLE }\left(k-1, \mathbf{r}^{k-1}, \widetilde{\mathbf{e}}_{k-1}^{(i-l)}, v_{1}, v_{2}, \gamma\right)
\end{aligned}
$$

6. Interpolation of the obtained correction $\widetilde{\mathbf{e}}_{k-1}^{(y)}$ to the finer grid (level $k$ )

$$
\mathbf{e}^{k} \leftarrow \mathrm{P}_{k-l}^{k} \widetilde{\mathbf{e}}_{k-l}^{(\gamma)},
$$


where $\mathrm{P}_{k-1}^{k}$ is an interpolation operator from coarser to finer grid.

7. Correction

$$
\hat{\mathbf{v}}_{k}^{(l)} \leftarrow \widetilde{\mathbf{v}}_{k}^{(0)}+\mathbf{e}^{k}
$$

8. (Post-relaxation). Perform $v_{2}$ additional smoothing iterations by

$$
\mathbf{v}_{k}^{(l)} \leftarrow \mathrm{S}_{\mathrm{k}}^{\mathcal{V}_{2}} \hat{\mathbf{v}}_{k}^{(l)}
$$

9. Return the result $\mathbf{v}_{k}^{(l)}$.

\section{End}

Full Multigrid Algorithm can be formulated as follows.

Algorithm FMG_CYCLE $\left(v_{1}, v_{2}, \gamma, \mu_{k}\right)$

$/ *$ Algorithm for approximate solution of a system of linear equations $\mathbf{A}_{K \max } \mathbf{v}_{K \max }=\mathbf{f}_{K \max }$ on the highest grid level $K_{\max }$. Parameters $v_{1}, \quad v_{2}, \quad \gamma$ are the same as in algorithm CGC_CYCLE, $\mu_{k}$ is a number of CGC-cycles performed on the grid level $k . * /$

\section{Begin}

1. Solve the system (15) by direct method on the grid level $k=0$. As a result we obtain the value $\mathbf{v}_{0}^{(l)}$.

For $k=1$ to $K_{\max }$ do

Begin

2. Interpolation of the obtained approximate solution $\mathbf{v}_{k-1}^{(I)}$ to the finer grid:

$$
\mathbf{v}_{k}^{(0)} \leftarrow \mathrm{P}_{k-l}^{k} \mathbf{v}_{k-1}^{(l)}
$$

Interpolation operator may be differ from one used in CGC-cycle.

3. Refining of the solution on the grid level $k$.

$$
\mathbf{v}_{k}^{(l)} \leftarrow \mathbf{v}_{k}^{(0)}
$$

For $i \leftarrow 1$ to $\mu_{k}$ do $\mathbf{v}_{k}^{(l)} \leftarrow$ CGC_CYCLE $\left(k, \mathbf{f}_{\mathbf{k}}, \mathbf{v}_{k}^{(l)}, v_{1}\right.$, $\left.v_{2}, \gamma\right)$

End

4. Return the result $\mathbf{v}_{K \max }^{(l)}$.

End

The main goal of the FMG-algorithm is to obtain an approximate solution $\mathbf{v}_{K \max }^{(l)}$, that satisfied

$$
\left\|\mathbf{v}_{K \max }-\mathbf{v}_{K \max }^{(l)}\right\| \leq \operatorname{ch}_{K_{\max }}^{2},
$$

where $\mathbf{v}_{\text {Kmax }}$ is the "exact" solution of (15) and $h_{\text {Kmax }}$ is a characteristic mesh-size on the highest grid level (we assume bilinear finite elements used, that provide the second order of approximation), constant $c$ is independent on the mesh-size.

Although the theory promises $\mathrm{O}\left(N_{\text {Kmax }}\right)$ complexity of the FMG-algorithm for achieving (24) for wide classes of problem, in practice it's not easy to get such efficiency for a specific problem. It may be related with incorrect choice of the parameters for CGC- and/or FMG algorithms in problems where physical or geometric anisotropy is presented. Therefore, the issues of a suitable tuning of the multigrid algorithm components play a key role in successful application of this method.

In [16] the question of appropriate choice of the multigrid parameters for the boundary-value problem (1) for the multi-layered foundation is investigated. This problem has not physical but geometric anisotropy: the length of the roadbed area considered is larger than its width and height. The determined parameters of the Multigrid Algorithm are: type of the relaxation operator (16); interpolation and projection operators (20), (18); method for creating the coarse-grid matrix $\mathbf{A}_{k}$ on the levels $k<K_{\max }$, numbers $v_{1}, v_{2}$ of pre- and postrelaxation, number $\gamma$ of correction refinement procedures, numbers $\mu_{k}$ of précising steps in FMGalgorithm.

It has been established, that the best choice, taking into account computational work expenses, is as follows.

Relaxation scheme is block-wise Gauss-Seidel operator on vertical lines, $v_{1}=1, v_{2}=0, \gamma=1$ (such variation of the CGC-cycle calls "V-Cycle"), $\mu_{k}=3$. The rest of parameters is typical. This choice of parameters provides the standard $\mathrm{O}\left(N_{K \max }\right)$ multigrid efficiency.

Prospects for further research. The following areas of improvement of methods for assessing the diagnostic parameters of the roadbed are promising.

1. Because of significant importance of the "prior" information for perfect inverse problem solution analysis, the main directions for further research may concern the methods of involvement of the information obtained by another considered methods of diagnostic parameters estimation (georadation, seismic, etc.), creation of the detailed roadbed maps with such data and precise binding technique development (including geolocation methods).

2. Improvement of inverse problem solving approaches (including optimal discretization procedures for unknown parameters vector $\mathbf{m}$ ) for obtaining the best resolution.

3. Development of modern approximation technique (including neural networks etc.) for fast online interpretation of scanning data.

\section{References}

1. T. Butko, M. Muzykin, A. Prokhorchenko, H. Nesterenko, H. Prokhorchenko, Transport and Telecommunication Journal, 20 (3), 215 (2019)

2. A. Glikman, NTF "GEOFIZPROGNOZ" po zakazu stroitelnogo zhurnala, Electronic resource. Access mode:

https://www.newgeophys.spb.ru/ru/article/railways crash/

3. E. Nepomnyashih, K. Kirpichnikov, Diagnostika sostoyaniya zheleznodorozhnogo puti, ZabIZhT, (2012) 109 c.

4. V. Petrenko, D. Yampolskij, I. Svyatko, Nauka ta progres transportu, 4 (46) (2013)

5. J. Luchko, I. Kravec, Visnik Odeskoyi derzhavnoyi akademiyi budivnictva ta arhitekturi, 71 (2018)

6. A. Nikolaides, Highway Engineering Pavements, Materials and Control of Quality, International Standard Book Number-13: 978-1-4665-7997-2 (eBook - PDF), (2014)

7. P. Ullidtz, Elsevier Science Publishers B.V., (1987) 
8. G. Flintsch, S. Katicha, J. Bryce, B. Ferne, S. Nell, B. Diefenderfer. Assessment of Continuous Pavement Deflection Measuring Technologies, (2013)

9. G. Morosiuk, M. Riley, J.B. Odoki, The Highway Development and Management Series, 6, (2004)

10. A. Das, Analysis of Pavement Structures, International Standard Book Number-13: 978-14665-5856-4 (eBook - PDF), (2015)

11. G. Berkovic, E. Shafi, Advances in Optics and Photonics 4, 441-471 (2012)
12. A. Tarantola, Inverse Problem Theory, 0-89871572-5 (pbk.), (2005)

13. A. Trofimov, Visnik AMSU, Series Technical Science, 2 (52), 48 (2014)

14. A. Trofimov, J. Petrova, Visnik AMSU, Series Technical Science, 2 (54), 48 (2015)

15. U. Trottenberg; C. W. Oosterlee; A. Schüller, Multigrid, Academic Press, 978-0-12-701070-0, (2001)

16. A. Trofimov, Visnik UMSF, Series Technical Science, 1 (55), 119 (2016) 